\title{
The Three-Layer Concentric Model of Glioblastoma: Cancer Stem Cells, Microenvironmental Regulation, and Therapeutic Implications
}

\author{
Luca Persano, ${ }^{1}$ Elena Rampazzo, ${ }^{1}$ Alessandro Della Puppa, ${ }^{2}$ \\ Francesca Pistollato, ${ }^{1}$ and Giuseppe Basso ${ }^{1}$ \\ ${ }^{1}$ Oncohematology Laboratory, Department of Paediatrics, University of Padova, \\ Via Giustiniani 3, Padova 35128, Italy \\ ${ }^{2}$ Neurosurgery, Department of Neuroscience, University of Padova, Via Giustiniani 2, \\ Padova 35128, Italy
}

Received 28 July 2011; Accepted 29 September 2011

Academic Editor: Martin Gotte

Tumors arising in the central nervous system are thought to originate from a sub-population of cells named cancer stem cells (CSCs) or tumor initiating cells (TICs) that possess an immature phenotype, combined with self-renewal and chemotherapy resistance capacity. Moreover, in the last years, these cells have been identified in particular brain tumor niches fundamental for supporting their characteristics. In this paper, we report studies from many authors demonstrating that hypoxia or the so called "hypoxic niche" plays a crucial role in controlling CSC molecular and phenotypic profile. We recently investigated the relationship existing between Glioblastoma (GBM) stem cells and their niche, defining the theory of three-concentric layers model for GBM mass. According to this model, GBM stem cells reside preferentially within the hypoxic core of the tumour mass, while more differentiated cells are mainly localized along the peripheral and vascularized part of the tumour. This GBM model provides explanation of the effects mediated by the tumour microenvironment on the phenotypic and molecular regulation of GBM stem cells, describing their spatial distribution in the tumor bulk. Moreover, we discuss the possible clinical implications of the creation of this model for future GBM patient management and novel therapeutic strategies development.

KEYWORDS: Hypoxia, cancer stem cells, topographic distribution, chemotherapy resistance 


\section{HYPOXIC REGULATION OF NEURAL AND CANCER STEM CELLS}

In vertebrate organisms, $\mathrm{O}_{2}$ is essential for life, acting as the central energy source in oxidative cell metabolism [1]. However, the physiologically normoxic conditions for embryonic or adults human cells generally fall in the 2-9\% $\mathrm{O}_{2}(14.4-64.8 \mathrm{mmHg})$ range [2], substantially lower than the $20.8 \% \mathrm{O}_{2}$ $\left(156 \mathrm{mmHg}\right.$, at sea level) in the air we breathe. Tissue oxygen tension $\left(\mathrm{pO}_{2}\right)$ values are conserved among mammalian species central nervous system (CNS) but vary along diverse brain regions. Since brain is one of the most metabolically active tissues in the body, $\mathrm{pO}_{2}$ levels are tightly regulated and, in particular, these values range from as low as $0.55 \%(4.1 \mathrm{mmHg})$ in the midbrain to $8.0 \%(60 \mathrm{mmHg})$ in the pia [3]. This heterogeneity allows conceiving that cells adjacent to the arterial inflow (high $\mathrm{pO}_{2}$ ) display characteristics and capacities that are different from those located at the venous end (low $\mathrm{pO}_{2}$ ).

The role of lowered oxygen in the regulation of neural precursors proliferation and differentiation has been described by our group in the last years [4]. We demonstrated that $20 \%$ oxygen promotes CNS precursors mitotic arrest and terminal differentiation to an astrocytic fate, with an increase in the number of p21 positive cells, a cyclin-dependent kinase inhibitor induced by $\mathrm{p} 53$, and a direct effector of mitotic arrest. Conversely, lowered oxygen increases proliferation and viability of CD133 ${ }^{+}$neural stem cells and oligodendrocyte progenitors [4]. Accordingly, a previous work from Smith and colleagues underlined the role of redox state as a central modulator of the balance between self-renewal and differentiation in dividing glial precursor cells [5]. They showed that treatment of dividing murine oligodendrocyte precursor cells (OPCs) with prooxidant drugs was associated with diminished progenitor division and increased number of differentiated cells. In contrast, OPCs treated with antioxidant molecules exhibited enhanced proliferation, self-renewal, and a marked reduction in oligodendrocyte generation [5]. Besides maintaining stemness and self-renewal of neural precursors, low $\mathrm{pO}_{2}(3-5 \%)$ also promotes generation of specific neural lineages. Culture of rat neural crest stem cells in low $\mathrm{O}_{2}$ promotes their survival and differentiation into tyrosine hydroxylase (TH)p sympathoadrenal cells or THp dopaminergic neurons [6-9]. Thus, self-renewal and differentiation capacity may depend on the cell type and the percentage of oxygen.

Disruption of the regulatory mechanism that controls self-renewal is reliably involved in the genesis of cancer initiating stem-like cells [10]. This is probably what happens in the human brain where neogenesis of mature cells persists throughout adult life within discrete brain regions: the dentate gyrus of the hippocampus and the subventricular zone of the forebrain lateral ventricles [11], both regions characterized by the presence of quiescent stem cells and mitotically active progenitor cells. These cell populations might function as a source of cells for transformation, potentially giving rise to CSCs. The first evidence of the presence of cells with stem-like characteristics in human brain tumours was reported by Ignatova and coworkers, who isolated clonogenic neurosphere-forming precursors from postsurgery specimens of human GBM and medulloblastoma tumors [12]. These stem-like cells expressed both neuronal and astroglial markers of differentiation, together with several key determinants of neural stem cell phenotype. These findings confirmed that brain tumours contain transformed, undifferentiated neural cells that respond to the same factors that activate adult neural stem cells and indicate that these tumour-initiating cells have the ability to self-renew, proliferate, and express typical markers of stem cells, such as the cell surface marker CD133 [13]. One aspect of the microenvironment that differs in normal versus tumor tissue is $\mathrm{O}_{2}$ tension. In fact, in solid tumors, $\mathrm{O}_{2}$ tensions can range from physiological $2.5 \%-5.3 \%$ levels to pathological values below $0.1 \%$ in necrotic regions [14].

A milestone in understanding oxygen physiology was the identification in 1995 by Wang et al. of the hypoxia inducible factor (HIF) transcriptional complex [15], which is a key regulatory system of response to hypoxia at both local and systemic levels. Recent evidence shows that HIF-1 $\alpha$ is also overexpressed in many human cancers and has been associated with increased patient mortality [16-22]. Moreover, overactivity of HIF-1 $\alpha$ has been implicated in tumor progression [16, 23-25]. At present, it is considered that increased HIF-1 $\alpha$ expression in cancer is caused by intratumoural hypoxia [26]. Indeed, robust tumour growth requires the presence of a local vascular network that supplies both oxygen and nutrients to tumour cells. However, a highly proliferating mass of tumour cells develops faster than the vasculature, and tumour cells 
rapidly meet up with a poorly and/or aberrantly vascularized microenvironment deficient in oxygen, that is, hypoxic. Blood capillaries carry oxygen to tissues, but since oxygen has a diffusion limit, its concentration decreases as the distance from capillaries increases. Microscopic examination of solid tumours reveals the presence of expanding tumour cells in proximity to capillaries and central portion of necrotic regions. This gradient of cell viability parallels that of a decreasing gradient of oxygen, which is accompanied by an increase in HIF- $1 \alpha$ levels, a decrease in the extracellular $\mathrm{pH}$, and an induction of resistance to radio- and chemotherapy [27]. Given these points, we can assume that HIF-1 $\alpha$ correlates with cancer aggressiveness and progression. According to the hypoxic tumour mass model, there are five main mechanisms by which enhancement of HIF-1 $\alpha$ levels may occur in cancer: (1) cancer cells have a reduced $\mathrm{O}_{2}$ metabolism via oxphos and an increased $\mathrm{O}_{2}$ metabolism via ROS generation, two situations that can activate HIF- $1 \alpha$ and frequently observed in cancer [28]; (2) cancer cells depend on glycolysis for keeping adequate ATP levels and glycolysis activation required an increase in HIF-1 $\alpha$ activity [29]; (3) cancer cells seem to have increased levels of $\mathrm{H}_{2} \mathrm{O}_{2}$, which is known to stabilize HIF-1 $\alpha$ [30]; (4) cancers display increased HIF$1 \alpha$ levels because of genetic alterations in the pathways responsible for HIF- $1 \alpha$ synthesis and degradation [26, 31]; (5) HIF- $1 \alpha$ stabilization is driven by low $\mathrm{pO}_{2}$ (low or absent vascularization) in solid tumors [32].

Many studies have been delineating the relationship of HIF- $1 \alpha$ signalling with a series of other pathways possibly involved in tumor progression. Gustafsson and coworkers showed direct correlation between Notch intracellular domain (NICD) and HIF-1 $\alpha$ demonstrating for the first time that hypoxia increases the stability of NICD through a direct interaction with the N-terminal region of HIF-1 $\alpha$, thus activating Notch signalling and maintaining cells in an undifferentiated state [33]. Recently, Land and colleagues demonstrated the involvement of HIF- $1 \alpha$ also in the Mammalian target of rapamycin (mTOR) signalling pathway [34]. mTOR positively enhances levels of HIF- $1 \alpha$-mediated transcription during conditions that favoured HIF- $1 \alpha$ stabilization, that is, hypoxia. Moreover, our group previously showed a correlation between hypoxia and BMP pathway modulation [4]. We observed that human neural precursors derived from $\mathrm{SVZ}$, when maintained under hypoxia $\left(5 \% \mathrm{O}_{2}\right)$, are less sensitive to BMP treatment in vitro, compared with cells maintained at $20 \% \mathrm{O}_{2}$. Given this, we hypothesized that oxygen could regulate BMP signalling at multiple steps, including SMAD activation and transcriptional induction of target genes such as Id1. A subsequent work from our group showed a counteracting effect of HIF-1 $\alpha$ and BMP signalling regulation in GBM [35]. Taken together, all these aspects show a marked involvement of hypoxia and HIF$1 \alpha$ in regulating not only physiological neural stem cells but also cancer progression and CSC maintenance.

\section{THE THREE-LAYER CONCENTRIC MODEL OF GLIOBLASTOMA}

In light of the pivotal role of the niche in controlling fate determination of normal stem cells, it has been suggested the existence of a CSC niche with the ability of modulating phenotype of the self-renewing/more immature tumor cell population [36]. In the effort to investigate the distribution of different tumor cell types within GBM tumor, we recently formulated the hypothesis of a multilayer model of GBM mass. GBM is the most frequent and most malignant brain cancer in adults [37], and despite the progress in defining the molecular characteristics of gliomas and the effort in formulating new therapeutic approaches, only marginal improvements have been reported for patients outcome [38]. GBM are highly proliferative and heterogeneous tumors and their morphology shows a great variety in a given patient, and from patient to patient [39]. Moreover, GBMs frequently display areas of necrosis that occurs in avascular low-oxygenated regions, and cells residing in the tumor bulk could bear different grades of differentiation [40]. Since the finding of a subpopulation of immature cells with stem-like properties in many types of solid tumors including GBM, that have been pointed as responsible for tumor onset and relapse [41, 42], we and many other researchers are trying to elucidate the relationship between differential tumor microenvironments and CSCs regulation [4, 36, 43-48]. Moreover, increasing evidence suggests that different nontumor cells and factors derived from the tumor microenvironment are necessary to regulate CSCs. Carcinoma-derived prostate fibroblasts, showing distinct molecular features from those that reside in normal tissues, were able to induce tumor progression in prostate epithelial cells $[49,50]$. Moreover, specialized microenvironments 
appear to be required for the engraftment of leukemic stem cells. Acute myeloid leukaemia cells have been shown to stick to extracellular matrix components, and CD44 has been reported to be fundamental for this process [51-53]. Also endothelial cells (ECs) seem to play a crucial function in creating a specific niche for CSCs residing in solid tumors, in particular of brain cancers [44, 54]. Since Calabrese and colleagues showed that putative brain tumor stem cells, expressing CD133 and Nestin, were localized near the capillaries and interacted with them in vitro, it is conceivable to hypothesize that CSCs require signals derived from the heterotypic interaction between endothelial cells (or other cellular entities) and the tumor epithelial compartment possibly involving Notch signalling, as described also for T-ALL xenografts [44, 55, 56]. In particular, ECs release soluble factors such as BDNF [57], PEDF [58], and VEGF-C [59] that enhance glioma CSCs proliferation and stemness maintenance. Confirming this hypothesis, a recent work demonstrated that the presence of immature, nestin-expressing cells in the perivascular niche is driven by activated Notch signaling in brain tumour precursor cells. This activation is mediated by eNOS enzyme [60] and by DLL4, a notch ligand overexpressed in ECs [61].

In this context of differential microenvironmental stimuli, possibly involved in the homing and regulation of CSCs, hypoxia seems to play a fundamental role in sustaining CSCs phenotype and aggressiveness, as described in the previous paragraph. One of the first attempts to clarify stem cells distribution in relation to tissue hypoxic gradients was discussed by Chow and coauthors, who developed a mathematical framework to evaluate $\mathrm{pO}_{2}$ distributions in the bone marrow hematopoietic compartment, considering the complex cellular architectures, cell layers, and clustering arrangements that compose bone marrow. They found that hematopoietic stem cells are located in regions with very low $\mathrm{pO}_{2}$ levels [62, 63], as previously suggested by Cipolleschi et al. in 1993 [64]. Subsequently, other studies confirmed this mathematical hypothesis describing the correlation between haematopoietic stem cell phenotype and regional hypoxia [65]. Authors showed that specific regions in the bone marrow with low blood perfusion levels contained the highest concentration of hematopoietic precursors and stem cells, according to an oxygen perfusion gradient [65]. HIF proteins via their stabilization under regionally defined hypoxia may thus serve as upstream regulators of many key processes involved in stem cell function.

The first evidence of a determined spatial distribution of different stem cells in brain tumors has been reported by Piccirillo et al. who described the existence of diverse subpopulations of CSCs, sharing common progenitors, albeit being different at the genetic and functional levels in different locations of brain tumors [48]. In this study, cells were obtained from biopsies dissected from the core of the GBM mass and from the periphery of the tumor. These CSC populations displayed different growth kinetics, clonogenic index and tumor-initiating ability, suggesting a more differentiated phenotype for GBM cells derived from the peripheral regions of the mass [48]. In this study, authors correlated diverse functional behaviour with distinct sets of genetic abnormalities, even though it is conceivable to hypothesize that tumor tissue oxygenation levels could also cooperate to induce different cell phenotypes in multiple compartments, as previously shown [36, 66-70]. Nonetheless, previous studies suggested that hypoxia is linked to genomic instability and p53 suppression [71, 72], thus raising the possibility of a causative relationship between biopsy collection site and genomic alterations described in this study [48]. In a recent study, tumor biopsies were collected from locations displaying differential levels of hypoxia in the tumor bulk. Samples were selected from within the tumor and in nonfunctional areas from peritumor and surrounding host tissue according to T1 contrast-weighed volumetric MRI [73]. Authors suggest that specific regional hypoxia is strictly correlated to the induction of a differential proinflammatory signature in GBM stem cells [73].

Our group developed a tridimensional model of the GBM tumor mass based on the differential molecular and phenotypic cellular identities distributed along the hypoxic gradient in GBM tumors [74, 75]. In this study, we identified three layers in the GBM mass and classified them as core (necrotic), intermediate layer, and peripheral/hyper-vascularized tumor area. Samples were collected from each of the three layers by means of image-guided surgery. The intermediate area has been considered as the transition area in between the inner core and the peripheral area (contrast enhancing ring at MRI after gadolinium administration) (Figure 1). By exploiting this procedure, we were able to reproducibly collect GBM samples with distinct phenotypes and functional characteristics according to the hypoxic gradient in the GBM tumor mass. Since 


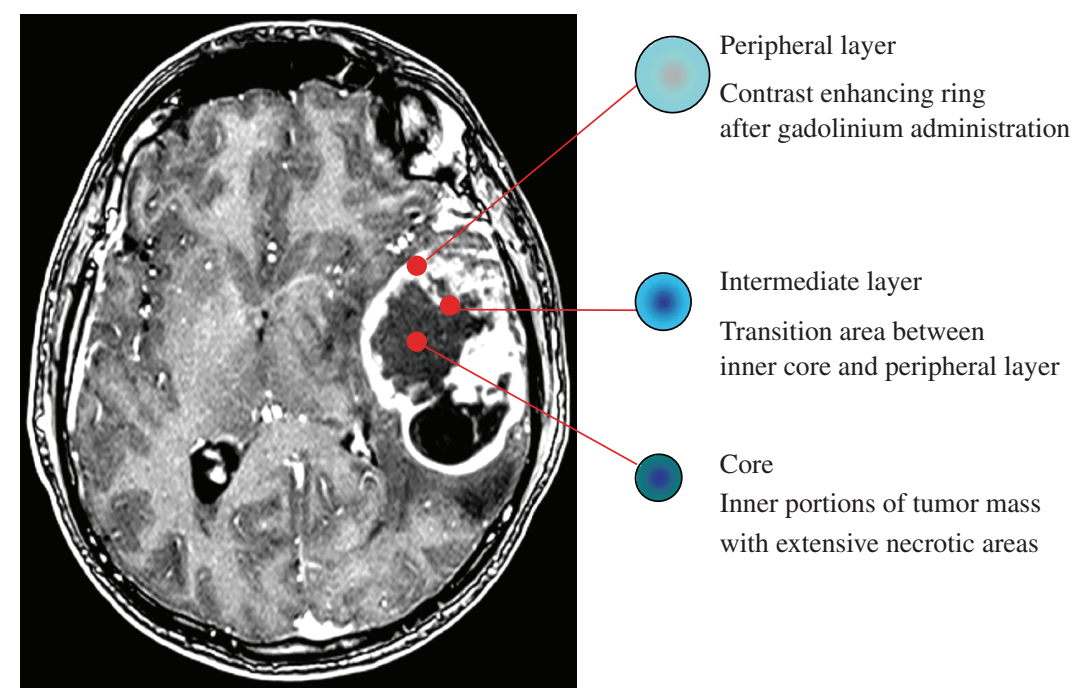

FIGURE 1: The three GBM layers identified by MRI analysis. Through radiological imaging we identified three layers in the GBM mass, classified as core, intermediate layer, and peripheral/hyper-vascularized tumor area. The intermediate area has been considered as the transition area in between the inner core and the peripheral layer (contrast enhancing ring). The surgical procedure was monitored by intraoperative imaging (neuronavigational setting). The neurosurgical resection of the peripheral regions had been done along the tumor/normal brain interface with the effort to avoid normal cells to be included in the specimen.

necrotic areas of solid tumors display a low-oxygenated microenvironment and cells lining necrotic tissues are exposed to milder hypoxia, it is conceivable to hypothesize a model in which more necrotic areas of GBM tissues are characterized by strong hypoxia and surrounded by less hypoxic cancer cells [14]. Oppositely cells from the contrast enhancing ring, supposed to be the most vascularized tumor layer, are exposed to the highest oxygen tension in the GBM tumor bulk. Although we did not directly measured oxygen tensions in the diverse location from which biopsies were collected, we found hypoxia markers such as HIF- $1 \alpha$ and its downstream targets CAIX and VEGF to be more abundant in the inner portions of the tumor mass, dramatically decreasing their expression in areas supposed to be more oxygenated [74] (Figure 2). Given the assumption that hypoxia promotes a stem cell phenotype of brain tumor cells [56, 70, 76-78], our data showed that more immature cells (expressing Nestin) were found in the inner core and intermediate layers, whereas more differentiated (as verified with neuronal and glial markers) were distributed at the periphery of the tumor [74, 75] (Figure 2). Despite the description of vascular stem cell niche for GBM [44], we found that $\mathrm{CD}_{133^{+}}$cancer stem cells were localized in the inner portions of the GBM mass, corroborating the hypothesized existence of a different stem cell niche in which cancer cells are exposed to very low levels of oxygen and activate, through HIF- $1 \alpha$ stabilization, a transcriptional program able to modulate the balance between proliferation and quiescence, aggressiveness, resistance to therapy, and invasiveness [26, 74, 79-81]. As a confirm, Seidel and colleagues reported that GBM-derived stem cells, identified using the side population (SP) approach and confirmed by CD133 staining, were highly enriched in both vascular and necrotic/hypoxic niches within the tumor bulk [82]. Moreover, they showed that hypoxia promoted the expression of a stem cell signature through HIF- $2 \alpha$-mediated upregulation of Oct-4 [82]. In 2006, Shinya Yamanaka's group demonstrated that the transcription factors Oct4, Sox2, Klf4, and c-Myc can convert a differentiated cell back to pluripotency over the course of a few weeks, thus reprogramming them into induced pluripotent stem (iPS) cells [83]. Thus hypoxia-dependent Oct-4 overexpression could play a fundamental role in determining tumor cell characteristics by pushing back more differentiated cells to a stem-like phenotype.

Although several data suggesting that more immature cells residing in the inner portions of the GBM mass could potentially be CSCs, further experiments will be needed to clearly characterize the functional 


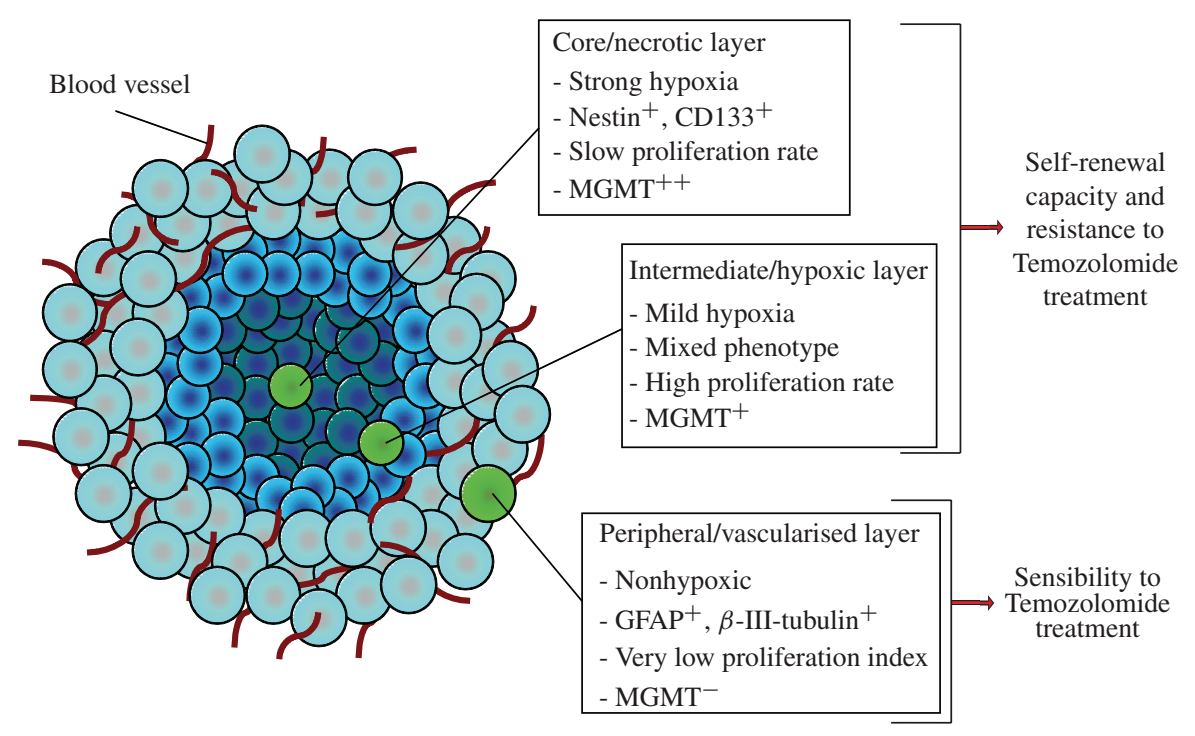

FIGURE 2: Phenotypic and molecular characteristics of the three concentric layers model of GBM. Inner portions of tumor mass display more immature cancer cells (Nestin ${ }^{+}$) and account for the highest percentages of cancer stem cells identified by CD133 staining. This identified stem cell population is resistant to alkylating agents-based chemotherapy in vitro. Peripheral GBM cells show a more differentiated phenotype (GFAP+, $\beta$-III-tubulin ${ }^{+}$) with low levels or absence of $\mathrm{CD}_{133^{+}}$cells. These peripheral tumor cells are highly sensitive to Temozolomide.

identity of these cells. In particular, according to the most recent definitions, a tumor cell can be considered as CSC after its tumorigenic potential has been evaluated in serial xenotransplantation experiments in mice. $\mathrm{CD}_{133^{+}}$cells derived from the different GBM layers must be tested for their tumorigenic potential before we can finally define these cells as CSC. Many authors described CD133 as a marker for the identification of GBM initiating cells and that $\mathrm{CD} 133^{+}$cells are able to generate tumors in mice $[84,85]$. Moreover, high levels of CD133 have been correlated to GBM tumor progression and dissemination [86]. Nevertheless, other studies reported that also the $\mathrm{CD} 133^{-}$subpopulation has a tumorigenic potential, raising some concerns about the real tumorigenic cell fraction of these tumors [87-90]. Even though we did not evaluate the tumor formation ability of GBM cells from different layers, cells located in the core and the intermediated layer show low levels of differentiation markers and high expression of Nestin [74], that has been reported to enhance the tumorigenic potential of GBM cells [91], suggesting a more aggressive phenotype of these cells.

This issue does not prevent us from pointing some remarks about the influence of tumor microenvironment in the regulation of CSCs. These data, resumed here from different studies, suggest that CSCs are located in specific microenvironments within the tumor, reminiscent of the stem cell niches described for neural stem cells [92, 93], and that tumors sustain their growth by the generation of new or expansion of preexisting niches, which support the maintenance of an undifferentiated phenotype. Given the important relation between stem cells and their niches [44, 74, 82], and the recent new definition of stemness described by Zipori, who suggested that stemness might be "a transient and reversible trait that almost any cell can assume given the correct niche" [94], we can argue that determining the precise location in which CSCs reside, besides defining their molecular characteristics, might help generating a new set of specific microenvironmental molecular targets. In this context, the definition of a multilayer model for GBM opens new questions on the right strategies to be developed in the future for GBM patients' management. In the next paragraph, therapeutic and surgical consequences of the generation of such a model will be discussed. 


\section{CLINICAL IMPLICATIONS}

One of the main pitfalls in cancer treatment is the hypoxia-associated resistance to photon radiotherapy, causative of tumour progression and relapse. The presence of molecular oxygen increases DNA damage through the formation of oxygen-free radicals, which occurs primarily after the interaction of radiation with intracellular water. Thus, because of this so-called "oxygen enhancement effect," the radiation dose required to achieve the same biologic effect is three times higher in the absence of oxygen than in the presence of normal oxygen levels. Evidence suggests that hypoxia-induced proteome and genome changes may also have a substantial impact on radioresistance by increasing the levels of heat shock proteins or by increasing the number of cells in a tumour with diminished apoptotic potential or increased proliferation rate, both of which have been linked to radioresistance [95].

Clinical implications arising from the three layers model of CSCs distribution in GBM could be remarkable, first of all, for diagnostic purposes. Since GBM displays different phenotypic patterns and molecular signalling activation in distinct regions (layers) of the tumor mass [74], the pathological characterization can be influenced by the site of sample collected by the surgeon throughout the tumor. For example, MGMT protein has been found differentially expressed among the three layers [74], and both MGMT protein expression and promoter methylation status are considered important prognostic factors $[96,97]$. This issue is fundamental because in the modern neuro-oncological setting, several diagnostic and prognostic markers [98] are commonly analyzed to predict tumor grade and the consequential therapeutic approach $[99,100]$. In addition, biomarkers are pivotal in the selection of glioma patients for their recruitment into clinical trials [101]. In this sense, site of the tumour sample collection could represent a remarkable bias for both selection and stratification of patients, as previously addressed by other authors [102].

The therapeutic implications of the three-layer model are more difficult to analyse because of the absence of clinical studies and the involvement of different variables. In this context, we are authorized to make only some reflections, whereas further studies are undoubtedly needed to confirm next considerations. However, the three-layer model could potentially have some interesting implications for both surgical and oncological settings. In fact, being putative CSCs mainly located in the core of GBM [74, 75], surgeon should achieve the complete removal of central region of tumor drastically reducing in this way the residual CSC population, which is considered the major responsible of resistance requiring supplementary treatments [103]. This assertion is apparently in contrast with the clinical experience of neurosurgeons that reports very high percentages of GBM recurrence, even after total removal of the primary tumor mass. There could be at least three different explanations for this phenomenon. First, we measured the presence of putative CSCs (CD133+ GBM cells), even if in very low numbers, also in the more peripheral regions of the tumor $[74,75]$. Second, hypoxia and HIF-1 $\alpha$ have been reported to enhance the migration properties of cancer cells by promoting metalloproteinase expression and migration-associated receptors such as CXCR4 $[104,105]$. Moreover, hypoxia has been described to regulate CXCR4-dependent cell migration also in lymphoma models [106]. It is conceivable to hypothesize that hypoxia increases GBM cell migratory ability, thus inducing cells residing in the core of the tumor to migrate through the above-described layers and potentially invade normal brain tissues outside of the GBM mass or form the scant $\mathrm{CD}_{133^{+}}$cell population of the peripheral layers [74]. Third, neural stem cells migrate from the subventricular zone or the dentate gyrus of the hippocampus to their final destination during the differentiation process in the developing and adult brain [107]. There could be the possibility that CSCs conserve their migration ability without being able to correctly differentiate toward a less proliferative and aggressive phenotype, infiltrating surrounding normal brain tissues.

During GBM management, surgery is followed by radiotherapy and concomitant alkylating agentsbased chemotherapy that could be virtually more effective against a tumoural residue possibly depleted of CSCs. A further consideration can be done about loco-regional therapies, which are treatments that surgeons can carry out directly in the surgical cave after tumour removal. This is the case of BCNU wafers that are a worldwide approved treatment for both newly diagnosed and recurrent high-grade gliomas [108]. They are constituted by degradable biopolymer wafers impregnated of Carmustine (an alkylating agent) 
that is released over few weeks in the surgical cave [109]. Wafers are implanted in the surgical cave after tumour removal, and they are positioned in tight contact with the brain surface infiltrated by tumour. When a complete removal of central core of tumour has been achieved, locoregional therapy such as BCNU wafers could be more effective against a limited CSC population. Finally, current chemotherapy points to tailored treatment. In this sense, these types of residual tumour cells should be targeted by supplementary treatments. When a large tumour exeresis is not achievable for different reasons (such as proximity to functional cortical areas and infiltration of major vessels), the residual tumour might be targeted by using prodifferentiating treatments together with conventional therapies, thus affecting CSC phenotype and aggressiveness.

\section{ACKNOWLEDGMENTS}

This work was supported by Fondazione Città della Speranza and by funds from the Italian Association for the Fight against Neuroblastoma (Pensiero Project), the Italian Association AIRC (Interregional pediatric project grant), and University of Padova, Young Investigators Grant.

\section{REFERENCES}

[1] J. Raymond and D. Segre, "The effect of oxygen on biochemical networks and the evolution of complex life," Science, vol. 311, no. 5768, pp. 1764-1767, 2006.

[2] M. C. Simon and B. Keith, "The role of oxygen availability in embryonic development and stem cell function," Nature Reviews Molecular Cell Biology, vol. 9, no. 4, pp. 285-296, 2008.

[3] R. D. Hoge and G. B. Pike, "Oxidative metabolism and the detection of neuronal activation via imaging," Journal of Chemical Neuroanatomy, vol. 22, no. 1-2, pp. 43-52, 2001.

[4] F. Pistollato, H. L. Chen, P. H. Schwartz, G. Basso, and D. M. Panchision, "Oxygen tension controls the expansion of human CNS precursors and the generation of astrocytes and oligodendrocytes," Molecular and Cellular Neuroscience, vol. 35, no. 3, pp. 424-435, 2007.

[5] J. Smith, E. Ladi, M. Mayer-Proschel, and M. Noble, "Redox state is a central modulator of the balance between self-renewal and differentiation in a dividing glial precursor cell," Proceedings of the National Academy of Sciences of the United States of America, vol. 97, no. 18, pp. 10032-10037, 2000.

[6] S. J. Morrison, M. Csete, A. K. Groves, W. Melega, B. Wold, and D. J. Anderson, "Culture in reduced levels of oxygen promotes clonogenic sympathoadrenal differentiation by isolated neural crest stem cells," Journal of Neuroscience, vol. 20, no. 19, pp. 7370-7376, 2000.

[7] A. Storch, G. Paul, M. Csete et al., "Long-term proliferation and dopaminergic differentiation of human mesencephalic neural precursor cells," Experimental Neurology, vol. 170, no. 2, pp. 317-325, 2001.

[8] L. Studer, M. Csete, S. H. Lee et al., "Enhanced proliferation, survival, and dopaminergic differentiation of CNS precursors in lowered oxygen," Journal of Neuroscience, vol. 20, no. 19, pp. 7377-7383, 2000.

[9] J. Milosevic, S. C. Schwarz, K. Krohn, M. Poppe, A. Storch, and J. Schwarz, "Low atmospheric oxygen avoids maturation, senescence and cell death of murine mesencephalic neural precursors," Journal of Neurochemistry, vol. 92, no. 4, pp. 718-729, 2005.

[10] A. L. Vescovi, R. Galli, and B. A. Reynolds, "Brain tumour stem cells," Nature Reviews Cancer, vol. 6, no. 6, pp. 425-436, 2006.

[11] P. S. Eriksson, E. Perfilieva, T. Bjork-Eriksson et al., "Neurogenesis in the adult human hippocampus," Nature Medicine, vol. 4, no. 11, pp. 1313-1317, 1998.

[12] T. N. Ignatova, V. G. Kukekov, E. D. Laywell, O. N. Suslov, F. D. Vrionis, and D. A. Steindler, "Human cortical glial tumors contain neural stem-like cells expressing astroglial and neuronal markers in vitro," GLIA, vol. 39, no. 3, pp. 193-206, 2002.

[13] S. Tamaki, K. Eckert, D. He et al., "Engraftment of sorted/expanded human central nervous system stem cells from fetal brain,” Journal of Neuroscience Research, vol. 69, no. 6, pp. 976-986, 2002.

[14] A. S. E. Ljungkvist, J. Bussink, J. H. A. M. Kaanders, and A. J. van der Kogel, "Dynamics of tumor hypoxia measured with bioreductive hypoxic cell markers," Radiation Research, vol. 167, no. 2, pp. 127-145, 2007. 
[15] G. L. Wang, B. H. Jiang, E. A. Rue, and G. L. Semenza, "Hypoxia-inducible factor 1 is a basic-helix-loophelix-PAS heterodimer regulated by cellular $\mathrm{O}_{2}$ tension," Proceedings of the National Academy of Sciences of the United States of America, vol. 92, no. 12, pp. 5510-5514, 1995.

[16] P. Birner, B. Gatterbauer, G. Oberhuber et al., "Expression of hypoxia-inducible factor- $1 \alpha$ in oligodendrogliomas: its impact on prognosis and on neoangiogenesis," Cancer, vol. 92, no. 1, pp. 165-171, 2001.

[17] A. W. Eckert, A. Schutze, M. H. W. Lautner, H. Taubert, J. Schubert, and U. Bilkenroth, "HIF-1 $\alpha$ is a prognostic marker in oral squamous cell carcinomas," International Journal of Biological Markers, vol. 25, no. 2, pp. 8792, 2010.

[18] J. R. Flynn, L. Wang, D. L. Gillespie et al., "Hypoxia-regulated protein expression, patient characteristics, and preoperative imaging as predictors of survival in adults with glioblastoma multiforme," Cancer, vol. 113, no. 5, pp. 1032-1042, 2008.

[19] X. Liang, M. Zheng, J. Jiang, G. Zhu, J. Yang, and Y. Tang, "Hypoxia-inducible factor-1 alpha, in association with TWIST2 and SNIP1, is a critical prognostic factor in patients with tongue squamous cell carcinoma," Oral Oncology, vol. 47, pp. 92-97, 2010.

[20] M. Z. Qiu, B. Han, H. Y. Luo et al., "Expressions of hypoxia-inducible factor- $1 \alpha$ and hexokinase-II in gastric adenocarcinoma: the impact on prognosis and correlation to clinicopathologic features," Tumor Biology, vol. 32, pp. 159-166, 2010.

[21] L. M. S. Seeber, N. Horree, P. van der Groep, E. van der Wall, R. H. M. Verheijen, and P. J. van Diest, "Necrosis related HIF- $1 \alpha$ expression predicts prognosis in patients with endometrioid endometrial carcinoma," BMC Cancer, vol. 10, article 307, 2010.

[22] J. Szkandera, G. Knechtel, M. Stotz et al., "Association of hypoxia-inducible factor 1-alpha gene polymorphisms and colorectal cancer prognosis," Anticancer Research, vol. 30, no. 6, pp. 2393-2397, 2010.

[23] D. L. Gillespie, K. Whang, B. T. Ragel, J. R. Flynn, D. A. Kelly, and R. L. Jensen, "Silencing of hypoxia inducible factor- $1 \alpha$ by RNA interference attenuates human glioma cell growth in vivo," Clinical Cancer Research, vol. 13, no. 8, pp. 2441-2448, 2007.

[24] L. Holmquist-Mengelbier, E. Fredlund, T. Lofstedt et al., "Recruitment of HIF- $1 \alpha$ and HIF- $2 \alpha$ to common target genes is differentially regulated in neuroblastoma: HIF- $2 \alpha$ promotes an aggressive phenotype," Cancer Cell, vol. 10, no. 5, pp. 413-423, 2006.

[25] G. L. Semenza, "Involvement of hypoxia-inducible factor 1 in human cancer," Internal Medicine, vol. 41, no. 2, pp. 79-83, 2002.

[26] G. L. Semenza, “Targeting HIF-1 for cancer therapy,” Nature Reviews Cancer, vol. 3, no. 10, pp. 721-732, 2003.

[27] P. Vaupel, "The role of hypoxia-induced factors in tumor progression," Oncologist, vol. 9, no. 5, pp. 10-17, 2004.

[28] M. López-Lázaro, "Does hypoxia really control tumor growth?” Cellular Oncology, vol. 28, no. 5-6, pp. $327-$ $329,2006$.

[29] R. H. Xu, H. Pelicano, Y. Zhou et al., "Inhibition of glycolysis in cancer cells: a novel strategy to overcome drug resistance associated with mitochondrial respiratory defect and hypoxia," Cancer Research, vol. 65, no. 2, pp. 613-621, 2005.

[30] N. S. Chandel, D. S. McClintock, C. E. Feliciano et al., "Reactive oxygen species generated at mitochondrial complex III stabilize hypoxia-inducible factor- $1 \alpha$ during hypoxia: a mechanism of $\mathrm{O}_{2}$ sensing," Journal of Biological Chemistry, vol. 275, no. 33, pp. 25130-25138, 2000.

[31] G. L. Semenza, "Hypoxia-inducible factor 1 and cancer pathogenesis," IUBMB Life, vol. 60, no. 9, pp. 591-597, 2008.

[32] P. H. Maxwell, G. U. Dachs, J. M. Gleadle et al., "Hypoxia-inducible factor-1 modulates gene expression in solid tumors and influences both angiogenesis and tumor growth," Proceedings of the National Academy of Sciences of the United States of America, vol. 94, no. 15, pp. 8104-8109, 1997.

[33] M. V. Gustafsson, X. Zheng, T. Pereira et al., "Hypoxia requires Notch signaling to maintain the undifferentiated cell state," Developmental Cell, vol. 9, no. 5, pp. 617-628, 2005.

[34] S. C. Land and A. R. Tee, "Hypoxia-inducible factor $1 \alpha$ is regulated by the mammalian target of rapamycin (mTOR) via an mTOR signaling motif," Journal of Biological Chemistry, vol. 282, no. 28, pp. 20534-20543, 2007. 
[35] F. Pistollato, H. L. Chen, B. R. Rood et al., "Hypoxia and HIF1 $\alpha$ repress the differentiative effects of BMPs in high-grade glioma," Stem Cells, vol. 27, no. 1, pp. 7-17, 2009.

[36] J. B. Sneddon and Z. Werb, "Location, location, location: the cancer stem cell niche," Cell Stem Cell, vol. 1, no. 6, pp. 607-611, 2007.

[37] T. Denysenko, L. Gennero, M. A. Roos et al., "Glioblastoma cancer stem cells: heterogeneity, microenvironment and related therapeutic strategies," Cell Biochemistry and Function, vol. 28, no. 5, pp. 343-351, 2010.

[38] R. Stupp, W. P. Mason, M. J. van Den Bent et al., "Radiotherapy plus concomitant and adjuvant temozolomide for glioblastoma," The New England Journal of Medicine, vol. 352, no. 10, pp. 987-996, 2005.

[39] M. Hassler, S. Seidl, B. Fazeny-Doerner et al., "Diversity of cytogenetic and pathohistologic profiles in glioblastoma," Cancer Genetics and Cytogenetics, vol. 166, no. 1, pp. 46-55, 2006.

[40] Z. Huang, L. Cheng, O. A. Guryanova, Q. Wu, and S. Bao, "Cancer stem cells in glioblastoma-molecular signaling and therapeutic targeting," Protein Cell, vol. 1, pp. 638-655, 2010.

[41] R. Galli, E. Binda, U. Orfanelli et al., "Isolation and characterization of tumorigenic, stem-like neural precursors from human glioblastoma," Cancer Research, vol. 64, no. 19, pp. 7011-7021, 2004.

[42] S. K. Singh, C. Hawkins, I. D. Clarke et al., "Identification of human brain tumour initiating cells," Nature, vol. 432, no. 7015, pp. 396-401, 2004.

[43] R. J. Gilbertson and J. N. Rich, "Making a tumour's bed: glioblastoma stem cells and the vascular niche," Nature Reviews Cancer, vol. 7, no. 10, pp. 733-736, 2007.

[44] C. Calabrese, H. Poppleton, M. Kocak et al., "A perivascular niche for brain tumor stem cells," Cancer Cell, vol. 11, no. 1, pp. 69-82, 2007.

[45] J. M. Heddleston, Z. Li, J. D. Lathia, S. Bao, A. B. Hjelmeland, and J. N. Rich, "Hypoxia inducible factors in cancer stem cells," The British Journal of Cancer, vol. 102, no. 5, pp. 789-795, 2010.

[46] J. M. Heddleston, Z. Li, R. E. McLendon, A. B. Hjelmeland, and J. N. Rich, "The hypoxic microenvironment maintains glioblastoma stem cells and promotes reprogramming towards a cancer stem cell phenotype," Cell Cycle, vol. 8, no. 20, pp. 3274-3284, 2009.

[47] Z. Li, S. Bao, Q. Wu et al., "Hypoxia-inducible factors regulate tumorigenic capacity of glioma stem cells," Cancer Cell, vol. 15, no. 6, pp. 501-513, 2009.

[48] S. G. M. Piccirillo, R. Combi, L. Cajola et al., "Distinct pools of cancer stem-like cells coexist within human glioblastomas and display different tumorigenicity and independent genomic evolution," Oncogene, vol. 28, no. 15, pp. 1807-1811, 2009.

[49] A. F. Olumi, G. D. Grossfeld, S. W. Hayward, P. R. Carroll, T. D. Tlsty, and G. R. Cunha, "Carcinoma-associated fibroblasts direct tumor progression of initiated human prostatic epithelium," Cancer Research, vol. 59, no. 19, pp. 5002-5011, 1999.

[50] J. B. Sneddon, H. H. Zhen, K. Montgomery et al., "Bone morphogenetic protein antagonist gremlin 1 is wideley expressed by cancer-associated stromal cells and can promote tumor cell proliferation," Proceedings of the National Academy of Sciences of the United States of America, vol. 103, no. 40, pp. 14842-14847, 2006.

[51] F. De Toni, C. Racaud-Sultan, G. Chicanne et al., "A crosstalk between the Wnt and the adhesion-dependent signaling pathways governs the chemosensitivity of acute myeloid leukemia," Oncogene, vol. 25, no. 22, pp. 3113-3122, 2006.

[52] L. Jin, K. J. Hope, Q. Zhai, F. Smadja-Joffe, and J. E. Dick, “Targeting of CD44 eradicates human acute myeloid leukemic stem cells," Nature Medicine, vol. 12, no. 10, pp. 1167-1174, 2006.

[53] D. S. Krause, K. Lazarides, U. H. von Andrian, and R. A. Van Etten, "Requirement for CD44 in homing and engraftment of BCR-ABL-expressing leukemic stem cells," Nature Medicine, vol. 12, no. 10, pp. 1175-1180, 2006.

[54] D. Hambardzumyan, O. J. Becher, M. K. Rosenblum, P. P. Pandolfi, K. Manova-Todorova, and E. C. Holland, "PI3K pathway regulates survival of cancer stem cells residing in the perivascular niche following radiation in medulloblastoma in vivo," Genes and Development, vol. 22, no. 4, pp. 436-448, 2008.

[55] S. Indraccolo, S. Minuzzo, M. Masiero et al., "Cross-talk between tumor and endothelial cells involving the Notch3-DII4 interaction marks escape from tumor dormancy," Cancer Research, vol. 69, no. 4, pp. 1314-1323, 2009.

[56] F. Pistollato, E. Rampazzo, L. Persano et al., "Interaction of hypoxia-inducible factor- $1 \alpha$ and Notch signaling regulates medulloblastoma precursor proliferation and fate," Stem Cells, vol. 28, no. 11, pp. 1918-1929, 2010. 
[57] C. Leventhal, S. Rafii, D. Rafii, A. Shahar, and S. A. Goldman, "Endothelial trophic support of neuronal production and recruitment from the adult mammalian subependyma," Molecular and Cellular Neurosciences, vol. 13, no. 6, pp. 450-464, 1999.

[58] C. Ramirez-Castillejo, F. Sanchez-Sanchez, C. Andreu-Agullo et al., "Pigment epithelium-derived factor is a niche signal for neural stem cell renewal," Nature Neuroscience, vol. 9, pp. 331-339, 2006.

[59] B. Le Bras, M. J. Barallobre, J. Homman-Ludiye et al., "VEGF-C is a trophic factor for neural progenitors in the vertebrate embryonic brain," Nature Neuroscience, vol. 9, no. 3, pp. 340-348, 2006.

[60] N. Charles, T. Ozawa, M. Squatrito et al., "Perivascular nitric oxide activates notch signaling and promotes stem-like character in PDGF-induced glioma cells," Cell Stem Cell, vol. 6, no. 2, pp. 141-152, 2010.

[61] S. Takano, T. Yamashita, and O. Ohneda, "Molecular therapeutic targets for glioma angiogenesis," Journal of Oncology, Article ID 351908, 2010.

[62] D. C. Chow, L. A. Wenning, W. M. Miller, and E. T. Papoutsakis, "Modeling $\mathrm{pO}_{2}$ distributions in the bone marrow hematopoietic compartment. II. Modified Kroghian models," Biophysical Journal, vol. 81, no. 2, pp. 685-696, 2001.

[63] D. C. Chow, L. A. Wenning, W. M. Miller, and E. T. Papoutsakis, "Modeling pO2 distributions in the bone marrow hematopoietic compartment. I. Krogh's model," Biophysical Journal, vol. 81, no. 2, pp. 675-684, 2001.

[64] M. G. Cipolleschi, P. D. Sbarba, and M. Olivotto, "The role of hypoxia in the maintenance of hematopoietic stem cells," Blood, vol. 82, no. 7, pp. 2031-2037, 1993.

[65] K. Parmar, P. Mauch, J. A. Vergilio, R. Sackstein, and J. D. Down, "Distribution of hematopoietic stem cells in the bone marrow according to regional hypoxia," Proceedings of the National Academy of Sciences of the United States of America, vol. 104, no. 13, pp. 5431-5436, 2007.

[66] B. Annabi, Y. T. Lee, S. Turcotte et al., "Hypoxia promotes murine bone-marrow-derived stromal cell migration and tube formation," Stem Cells, vol. 21, no. 3, pp. 337-347, 2003.

[67] K. L. Covello, J. Kehler, H. Yu et al., "HIF-2 $\alpha$ regulates Oct-4: effects of hypoxia on stem cell function, embryonic development, and tumor growth," Genes and Development, vol. 20, no. 5, pp. 557-570, 2006.

[68] H. Iwasaki and T. Suda, "Cancer stem cells and their niche," Cancer Science, vol. 100, no. 7, pp. 1166-1172, 2009.

[69] H. Li, X. Fan, and J. Houghton, "Tumor microenvironment: the role of the tumor stroma in cancer," Journal of Cellular Biochemistry, vol. 101, no. 4, pp. 805-815, 2007.

[70] A. Soeda, M. Park, D. Lee et al., "Hypoxia promotes expansion of the CD133-positive glioma stem cells through activation of HIF-1 $\alpha$," Oncogene, vol. 28, no. 45, pp. 3949-3959, 2009.

[71] E. M. Hammond and A. J. Giaccia, "The role of p53 in hypoxia-induced apoptosis," Biochemical and Biophysical Research Communications, vol. 331, no. 3, pp. 718-725, 2005.

[72] M. Koshiji, K. K. To, S. Hammer et al., "HIF- $1 \alpha$ induces genetic instability by transcriptionally downregulating MutS $\alpha$ expression," Molecular Cell, vol. 17, no. 6, pp. 793-803, 2005.

[73] M. Tafani, M. Di Vito, A. Frati et al., "Pro-inflammatory gene expression in solid glioblastoma microenvironment and in hypoxic stem cells from human glioblastoma," Journal of Neuroinflammation, vol. 8, p. 32, 2011.

[74] F. Pistollato, S. Abbadi, E. Rampazzo et al., "Intratumoral hypoxic gradient drives stem cells distribution and MGMT expression in glioblastoma," Stem Cells, vol. 28, no. 5, pp. 851-862, 2010.

[75] F. Pistollato, L. Persano, A. D. Puppa, E. Rampazzo, and G. Basso, "Isolation and expansion of regionally defined human glioblastoma cells in vitro," Current Protocols in Stem Cell Biology, chapter 3, unit 3.4, 2011.

[76] E. E. Bar, A. Lin, V. Mahairaki, W. Matsui, and C. G. Eberhart, "Hypoxia increases the expression of stem-cell markers and promotes clonogenicity in glioblastoma neurospheres," The American Journal of Pathology, vol. 177, no. 3, pp. 1491-1502, 2010.

[77] J. Mazumdar, V. Dondeti, and M. C. Simon, "Hypoxia-inducible factors in stem cells and cancer," Journal of Cellular and Molecular Medicine, vol. 13, no. 11-12, pp. 4319-4328, 2009.

[78] F. Pistollato, E. Rampazzo, S. Abbadi et al., "Molecular mechanisms of HIF- $1 \alpha$ modulation induced by oxygen tension and BMP2 in glioblastoma derived cells," PLoS One, vol. 4, no. 7, Article ID e6206, 2009.

[79] B. Das, R. Tsuchida, D. Malkin, G. Koren, S. Baruchel, and H. Yeger, "Hypoxia enhances tumor stemness by increasing the invasive and tumorigenic side population fraction," Stem Cells, vol. 26, no. 7, pp. 1818-1830, 2008. 
[80] A. L. Harris, "Hypoxia—a key regulatory factor in tumour growth," Nature Reviews Cancer, vol. 2, no. 1, pp. 38-47, 2002.

[81] S. Diabira and X. Morandi, "Gliomagenesis and neural stem cells: key role of hypoxia and concept of tumor "neo-niche"," Medical Hypotheses, vol. 70, no. 1, pp. 96-104, 2008.

[82] S. Seidel, B. K. Garvalov, V. Wirta et al., "A hypoxic niche regulates glioblastoma stem cells through hypoxia inducible factor 2 alpha," Brain, vol. 133, pp. 983-995, 2010.

[83] K. Takahashi and S. Yamanaka, "Induction of pluripotent stem cells from mouse embryonic and adult fibroblast cultures by defined factors," Cell, vol. 126, no. 4, pp. 663-676, 2006.

[84] C. Altaner, "Glioblastoma and stem cells," Neoplasma, vol. 55, no. 5, pp. 369-374, 2008.

[85] A. Natsume, S. Kinjo, K. Yuki et al., "Glioma-initiating cells and molecular pathology: implications for therapy," Brain Tumor Pathology, vol. 28, pp. 1-12, 2011.

[86] X. Yan, L. Ma, D. Yi et al., "A CD133-related gene expression signature identifies an aggressive glioblastoma subtype with excessive mutations," Proceedings of the National Academy of Sciences of the United States of America, vol. 108, pp. 1591-1596, 2011.

[87] K. Nishide, Y. Nakatani, H. Kiyonari, and T. Kondo, "Glioblastoma formation from cell population depleted of prominin1-expressing cells," PLoS One, vol. 4, no. 8, Article ID e6869, 2009.

[88] A. T. Ogden, A. E. Waziri, R. A. Lochhead et al., "Identification of A2B5+CD133- tumor-initiating cells in adult human gliomas," Neurosurgery, vol. 62, no. 2, pp. 505-514, discussion 514-505, 2008.

[89] A. Sato, K. Sakurada, T. Kumabe et al., "Association of stem cell marker CD133 expression with dissemination of glioblastomas," Neurosurgical Review, vol. 33, no. 2, pp. 175-183, discussion 183-174, 2010.

[90] J. Wang, P. O. Sakariassen, O. Tsinkalovsky et al., "CD133 negative glioma cells form tumors in nude rats and give rise to CD133 positive cells," International Journal of Cancer, vol. 122, no. 4, pp. 761-768, 2008.

[91] L. Prestegarden, A. Svendsen, J. Wang et al., "Glioma cell populations grouped by different cell type markers drive brain tumor growth," Cancer Research, vol. 70, no. 11, pp. 4274-4279, 2010.

[92] Q. Shen, Y. Wang, E. Kokovay et al., "Adult SVZ stem cells lie in a vascular niche: a quantitative analysis of niche cell-cell interactions," Cell Stem Cell, vol. 3, no. 3, pp. 289-300, 2008.

[93] M. Tavazoie, L. Van der Veken, V. Silva-Vargas et al., "A specialized vascular niche for adult neural stem cells," Cell Stem Cell, vol. 3, no. 3, pp. 279-288, 2008.

[94] D. Zipori, "The nature of stem cells: state rather than entity," Nature Reviews Genetics, vol. 5, no. 11, pp. 873-878, 2004.

[95] A. M. Spence, M. Muzi, K. R. Swanson et al., "Regional hypoxia in glioblastoma multiforme quantified with $[18 \mathrm{~F}]$ fluoromisonidazole positron emission tomography before radiotherapy: correlation with time to progression and survival," Clinical Cancer Research, vol. 14, no. 9, pp. 2623-2630, 2008.

[96] M. E. Hegi, A. C. Diserens, S. Godard et al., "Clinical trial substantiates the predictive value of O-6-methylguanine-DNA methyltransferase promoter methylation in glioblastoma patients treated with temozolomide," Clinical Cancer Research, vol. 10, no. 6, pp. 1871-1874, 2004.

[97] M. E. Hegi, L. Liu, J. G. Herman et al., "Correlation of O6-methylguanine methyltransferase (MGMT) promoter methylation with clinical outcomes in glioblastoma and clinical strategies to modulate MGMT activity," Journal of Clinical Oncology, vol. 26, no. 25, pp. 4189-4199, 2008.

[98] M. Labussiere, X. W. Wang, A. Idbaih, F. Ducray, and M. Sanson, "Prognostic markers in gliomas," Future Oncology, vol. 6, no. 5, pp. 733-739, 2010.

[99] J. T. Huse, H. S. Phillips, and C. W. Brennan, "Molecular subclassification of diffuse gliomas: seeing order in the chaos," Glia, vol. 59, pp. 1190-1199, 2011.

[100] L. Karayan-Tapon, V. Quillien, J. Guilhot et al., "Prognostic value of O6-methylguanine-DNA methyltransferase status in glioblastoma patients, assessed by five different methods," Journal of Neuro-Oncology, vol. 97, no. 3 , pp. 311-322, 2010.

[101] D. A. Reardon, E. Galanis, J. F. DeGroot et al., "Clinical trial end points for high-grade glioma: the evolving landscape," Journal of Neuro-Oncology, vol. 13, pp. 353-361, 2011.

[102] V. T. Cao, T. Y. Jung, S. Jung et al., "The correlation and prognostic significance of MGMT promoter methylation and MGMT protein in glioblastomas," Neurosurgery, vol. 65, no. 5, pp. 866-875, 2009.

[103] L. Cheng, S. Bao, and J. N. Rich, "Potential therapeutic implications of cancer stem cells in glioblastoma," Biochemical Pharmacology, vol. 80, no. 5, pp. 654-665, 2010. 
[104] S. Coma, A. Shimizu, and M. Klagsbrun, "Hypoxia induces tumor and endothelial cell migration in a semaphorin 3F- and VEGF-dependent manner via transcriptional repression of their common receptor neuropilin 2," Cell Adhesion \& Migration, vol. 5, pp. 266-275, 2011.

[105] P. A. Cronin, J. H. Wang, and H. P. Redmond, "Hypoxia increases the metastatic ability of breast cancer cells via upregulation of CXCR4," BMC Cancer, vol. 10, article 225, 2010.

[106] E. Piovan, V. Tosello, S. Indraccolo et al., "Differential regulation of hypoxia-induced CXCR4 triggering during B-cell development and lymphomagenesis," Cancer Research, vol. 67, no. 18, pp. 8605-8614, 2007.

[107] A. Quinones-Hinojosa, N. Sanai, O. Gonzalez-Perez, and J. M. Garcia-Verdugo, "The human brain subventricular zone: stem cells in this niche and its organization," Neurosurgery Clinics of North America, vol. 18, pp. 15-20, 2007.

[108] S. H. Lin and L. R. Kleinberg, "Carmustine wafers: localized delivery of chemotherapeutic agents in CNS malignancies," Expert Review of Anticancer Therapy, vol. 8, no. 3, pp. 343-359, 2008.

[109] A. B. Fleming and W. M. Saltzman, "Pharmacokinetics of the carmustine implant," Clinical Pharmacokinetics, vol. 41, no. 6, pp. 403-419, 2002.

\section{This article should be cited as follows:}

Luca Persano, Elena Rampazzo, Alessandro Della Puppa, Francesca Pistollato, and Giuseppe Basso, “The Three-Layer Concentric Model of Glioblastoma: Cancer Stem Cells, Microenvironmental Regulation, and Therapeutic Implications," TheScientificWorldJOURNAL, vol. 11, pp. 1829-1841, 2011. 

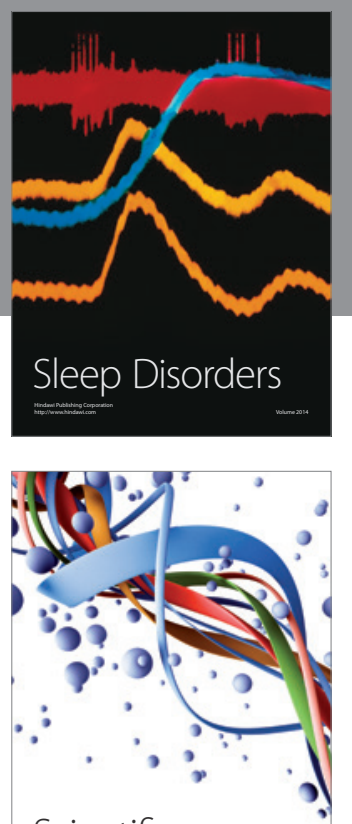

Scientifica
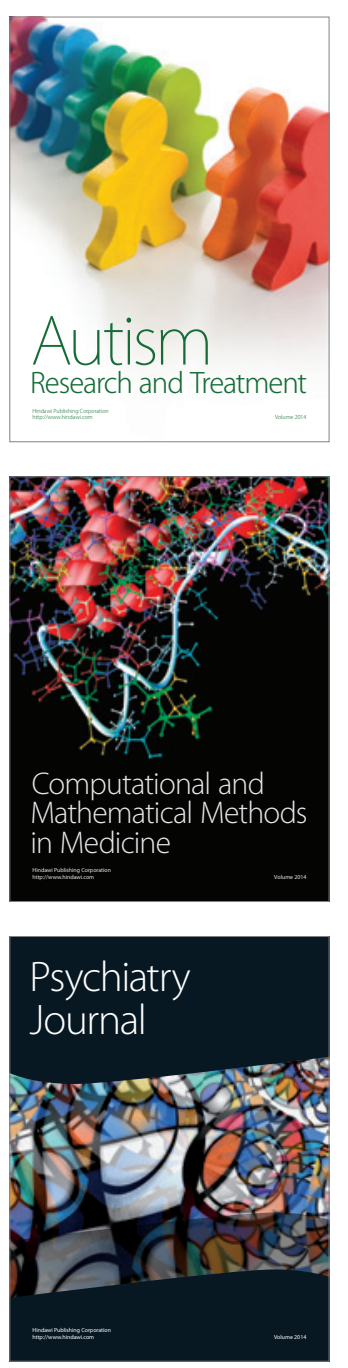
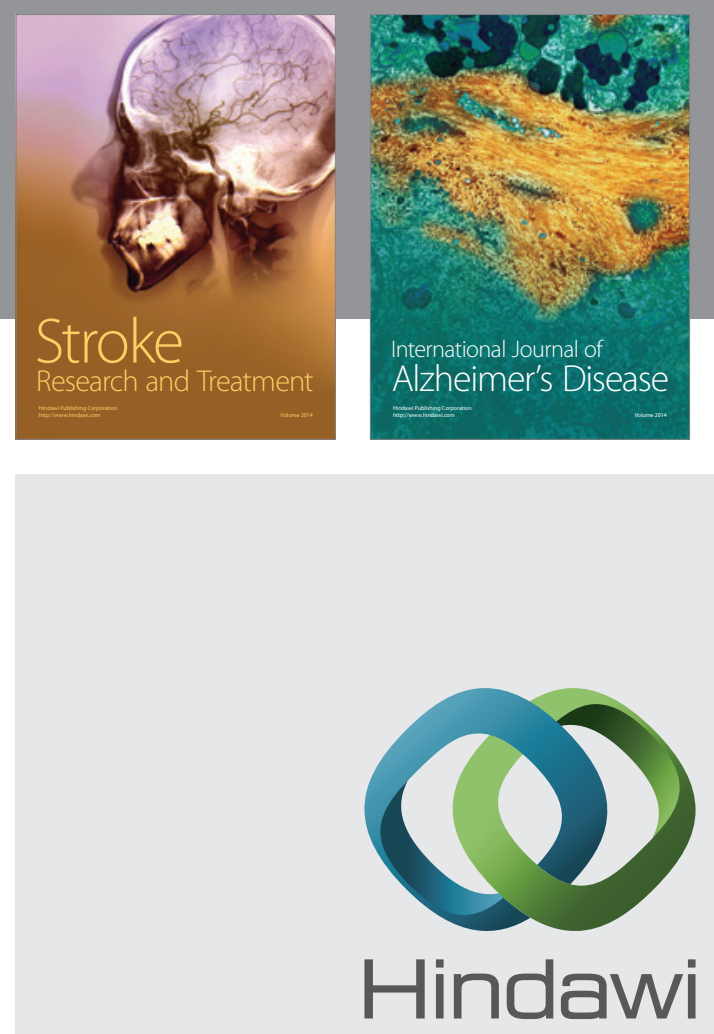

Submit your manuscripts at

http://www.hindawi.com
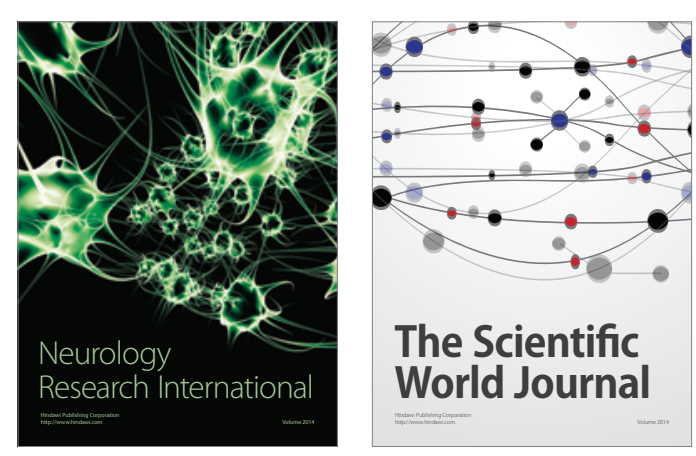

The Scientific World Journal

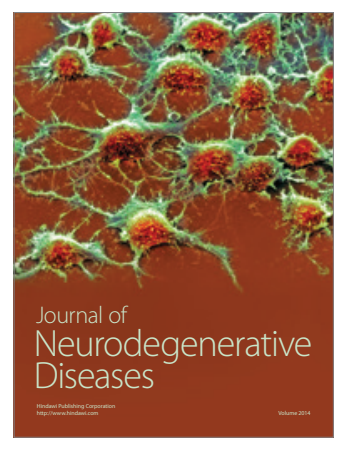

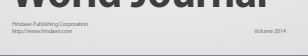

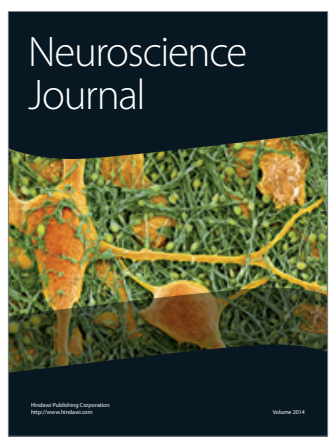

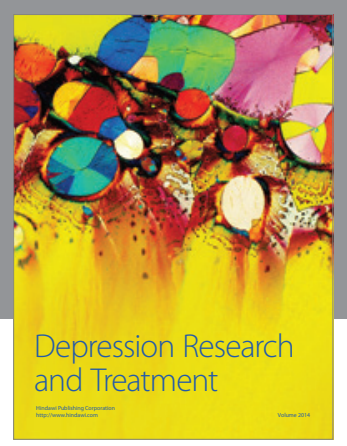
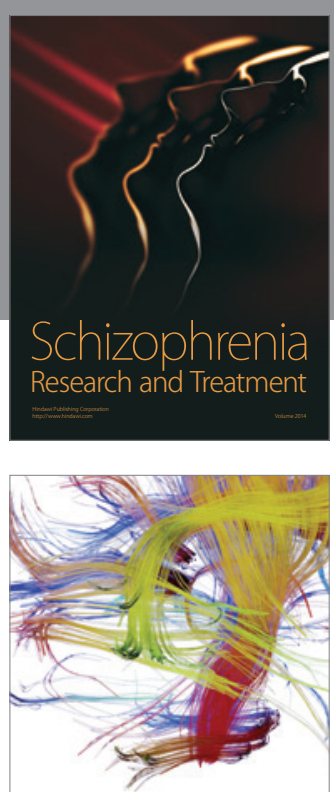

Brain Science

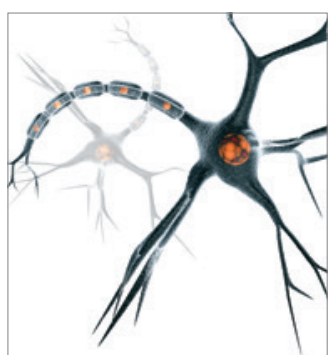

Neural Plasticity
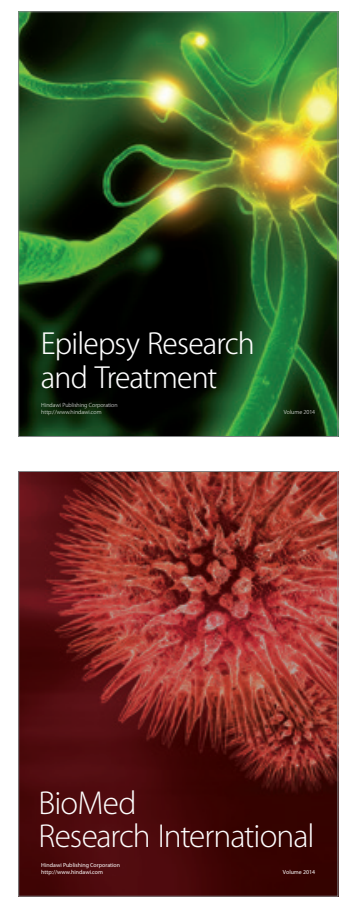

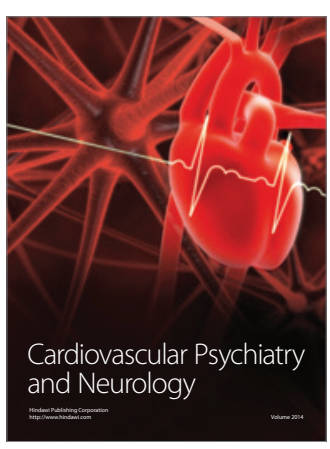

Parkinson's

Disease
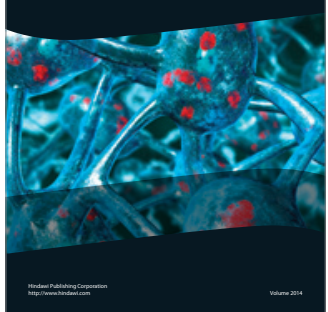